\title{
Encouraging Collaboration in Hybrid Therapy Games for Autistic Children
}

\author{
Sebastian Marwecki \\ Human-Computer Interaction Group \\ University of Konstanz \\ 78457 Konstanz, Germany \\ sebastian.marwecki@uni-konstanz.de \\ Roman Rädle \\ Human-Computer Interaction Group \\ University of Konstanz \\ 78457 Konstanz, Germany \\ roman.raedle@uni-konstanz.de

\section{Harald Reiterer} \\ Human-Computer Interaction Group \\ University of Konstanz \\ 78457 Konstanz, Germany \\ harald.reiterer@uni-konstanz.de
}

\begin{abstract}
Social competence and communicative skills of children with autism spectrum disorders are supported by behavioral therapy. "Serious games", especially

therapeutic games on a hybrid medium, have been proven to serve as a useful tool for behavioral therapy. In this work, we present such a hybrid therapy game:

"Invasion of the Wrong Planet". Based on the game, we demonstrate essential design principles for the development of therapeutic games. We focus on specific aspects of behavioral therapy, depending on which desired behavior is encouraged and not enforced. The project provides a basis for discussion on how collaboration using therapeutic games in general can be improved.
\end{abstract}

\section{Author Keywords}

Autism; health; behavior therapy; games; hybrid

interactive surfaces; CSCW

\section{ACM Classification Keywords}

H.5.3. Group and Organization Interfaces: Computersupported cooperative work.

\section{General Terms}

Design; Experimentation; Human Factors 


\section{Introduction}

Autism is a complex developmental disorder. Children with autism have impairments in social interaction and communicative skills and show stereotyped or repetitive behavior [2]. These impairments in social interaction may include a lack of understanding or inappropriate use of non-verbal behaviors. They also may include the inability to develop peer relationships or the lack of need to share interests or enjoyment with others. Communication skills are limited by the incapability to initiate or sustain conversations. Spoken language, if present at all, is delayed. The range of interests is narrowed to just a few topics or activities. The preoccupation with these interests is very stereotyped, inflexible, and often abnormally intense. Affected children also often have poor motor skills and coordination as well as impairments in their cognitive skills.

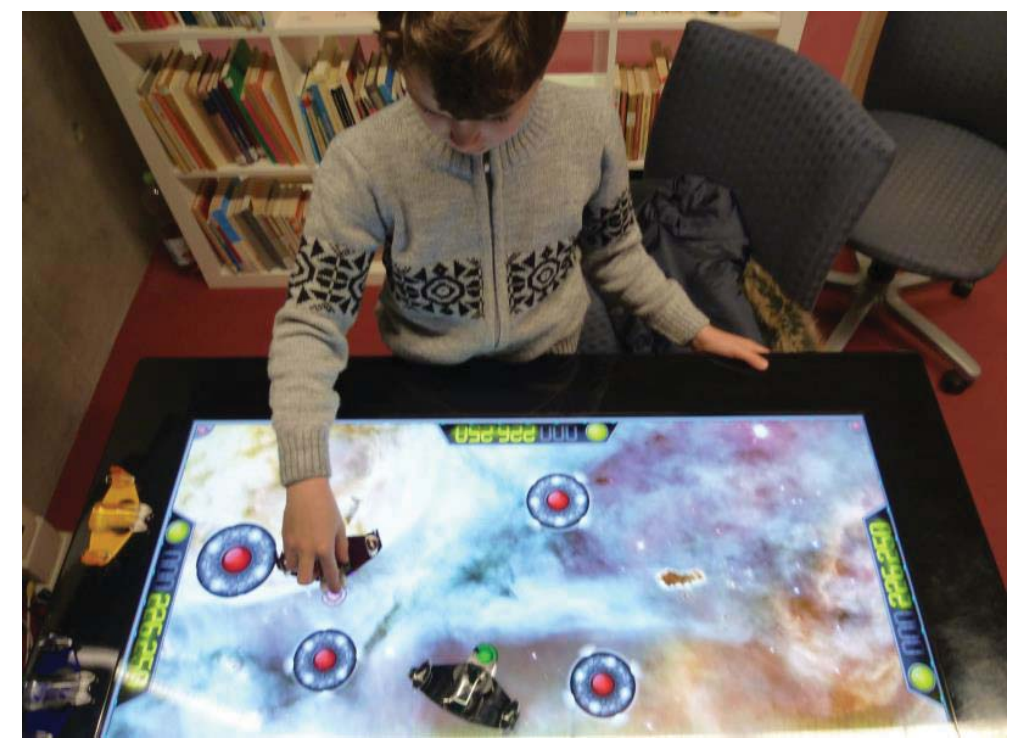

Figure 1. A child playing the game.
These impairments vary for each individual and can cover a wide range of manifestations, which is referred to as "autism spectrum disorders" (ASD). ASD can be recognized at all levels of intelligence. Depending on an intelligence threshold, the spectrum is often divided into low, medium, and high functioning autism (LFA MFA, HFA). Children with "Asperger syndrome" (AS) do not demonstrate such severe impairments in

communicative skills or limitation in cognitive abilities and can be compared with autistic children with HFA.

ASD are not curable. The main goals of therapy are to decrease the symptoms, help affected persons to accept their situation, and provide support for their families. Through behavior therapy, one can condition desired behaviors and develop strategies for overcoming his or her deficits. The "Treatment and Education of Autistic and Related Communication Handicapped Children" (TEACCH) [5] is based on such behavioral therapy. Intervention strategies are generally very structured and support the visual way of thinking, which is immanent to people with autism. One way to provide such a visual structure is through "therapeutic games". Since they are "serious games", these games have the potential to offer a high grade of intrinsic motivation and, at the same time, combine this motivation with an extrinsic gain. This gain, in this case a therapeutic effect, can be maximized by a hybrid medium, such as a hybrid interactive surface. Games on such a medium are often referred to as "hybrid games". Hybrid games have the means to blend together digital and analog advantages [4]. Players are motivated and feel secure in the digital setting [3]. At the same time, the form factor allows a face-to-face communication between the players. This combination of advantages can now be used for therapeutic games. 

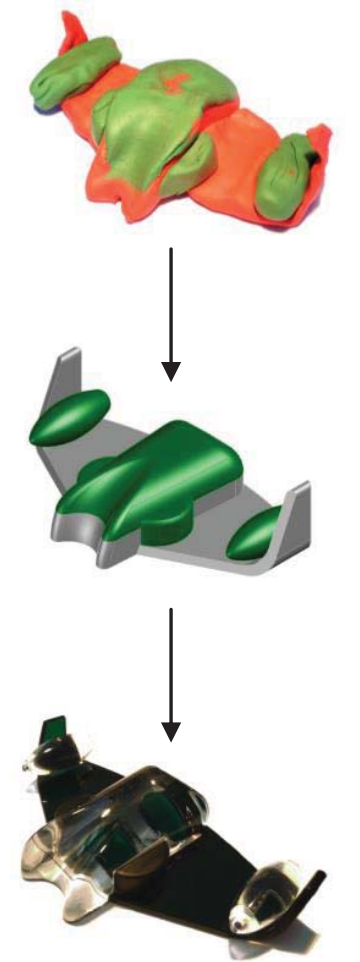

Figure 2. In the design process of the tokens, we used plasticine models, which were made by a child of the postulated age. Using these models, we created the computer model and the plexiglass model, respectively. The tokens are provided in four different colors (red, green, blue, yellow) and are $115 \times 68$ millimeters in size.

\section{Related Work}

The idea behind hybrid therapy games is not new. Recent studies $[3,6,7]$ have proven the potential of using hybrid interactive surfaces for therapy games to treat HFA and AS. Hybrid therapy games are an effective instrument for therapists in group therapies, and even more effective than common therapeutic games, as they provide a higher level of motivation and involvement. However, in said studies, players were forced into collaborative actions in order to foster social behavior and communication. This "enforced collaboration" (EC) [6] was proven to have a meaningful therapeutic effect. We believe that the design principle of EC can still be elaborated further. In prevalent behavioral therapies, desired behavior is never enforced, but encouraged and rewarded, while undesired behavior is penalized. Therapeutic games are an instrument of behavioral therapy. That being said, therapeutic games should provide the means to encourage collaboration, instead of enforcing it. A game that leads its players into voluntary collaborative actions may better aid in transferring social behavior and communicative skills into everyday life.

\section{Requirements and Implementation}

In order to show the importance of "encouraged collaboration" in therapeutic games, we developed a game, which serves as a basis for this discussion: "Invasion of the Wrong Planet". Based on a hybrid medium, the Samsung SUR40 with Microsoft PixelSense ${ }^{1}$, the game provides face-to-face communication and a possibility for social interaction within the comfortable and controllable digital setting.

\footnotetext{
${ }_{1}^{1} \mathrm{http://www.microsoft.com/en-us/pixelsense/default.aspx}$
}

To generate the requirements for the project, we worked together with two educational advisers for ASD and one therapist with several years of experience in the diagnosis and treatment of ASD. In the following, we list these eight requirements separately and describe how we strove to match them.

Requirement 1: The game should be designed for children with either HFA or AS between the ages of eight and twelve years. Since the prevalence for autism is significantly higher for boys than for girls, the narrative structure of the game should focus on boys.

In "Invasion of the Wrong Planet", the players have the task of defending the earth (the "wrong planet" ${ }^{2}$ ) from alien invaders. The narrative structure is supported by the design of the tokens (see Figure 2). The players can move their ship through space by moving the tokens across the screen. They can then shoot enemies by pressing the virtual button in front of their token. The familiarity of the setting is intentional, for it may provide a motivation similar to commercial games played at home on consoles or the computer. Also it reduces the cognitive affordance of the game contents. Nevertheless, the cognitive level is too high to be easily understood by children with LFA or MFA. The game serves as a motivator and basis for group discussions in group therapy sessions for children with HFA or AS. It should be mentioned that this project may only serve as a tool for group therapy; it is not meant to replace a therapist.

2 The name of the game is based on the term "wrong planet syndrome", an alternate description of ASD. Children, though normal and their surroundings as all the more odd. They feel like they are on the "wrong planet". 

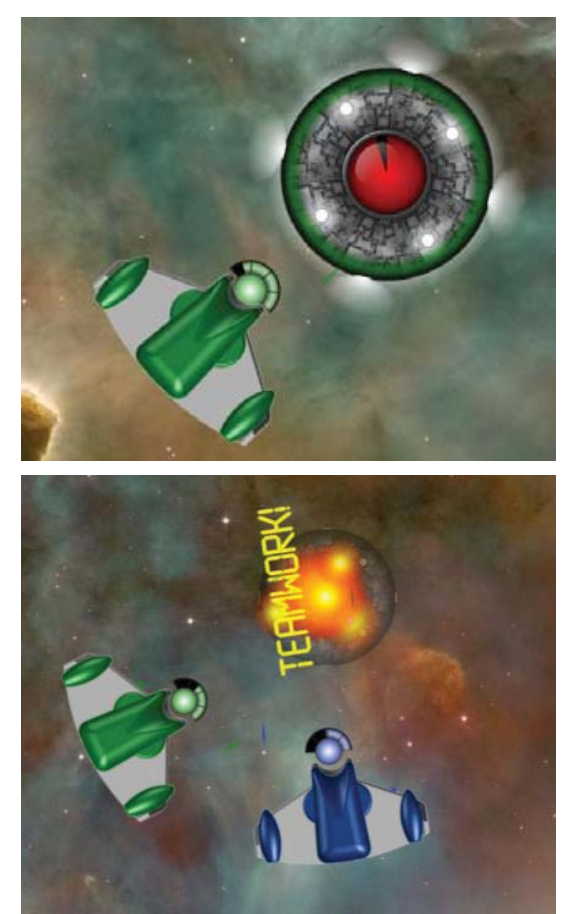

Figure 3. Some ships, called

"Raiders," can be destroyed by a single player. However, when multiple players agree to confront the enemy at the same time, the time required to eliminate the ship shrinks

exponentially and more points are given.
Requirement 2: Communication and social interaction must happen on a game-based level with relation to the goal of the game.

The game consists of different levels, i.e., solar systems, from which the players are allowed to choose. Depending on the level, the players are confronted with different tasks best to be solved collaboratively. These tasks consist of eliminating different alien ships together. Each of these enemy ships requires a different strategy, which the group has to figure out through discussion. Each strategy involves the players in collaborative actions. When the players eliminate an enemy ship, they collect points.

Requirement 3: The game should encourage collaborative behavior of the players, but not enforce it. Collaboration on the part of multiple players should therefore be rewarded more than the actions of one individual player. Feedback should be provided in a timely manner and condition the desired behavior(s).

Utilizing the concept of encouraged collaboration, all elements encourage communication and social behavior. A single player can eliminate each enemy. However, players who act collaboratively will achieve a significantly higher score. For examples of this, see Figures $3-8$.

The game also provides visual and acoustic feedback when the players receive a higher score through collaboration. Due to the strong audiovisual feedback, players receive an immediate response to their actions and are motivated into collaborative behavior.
Requirement 4: The progress, structure, and goal of the game should be clear and easy to understand. This is best done in a visualized and structured manner similar to the TEACCH approach.

The game offers the possibility of an explanation, a tutorial, when a new game element appears in the game. We tried to follow the TEACCH approach and used visual explanation wherever possible. The time remaining is visualized in a pie chart next to the score display. The game provides audiovisual feedback after each of a player's actions.

Requirement 5: The game should not penalize players limited in playing skills, that is to say cognitive and motor skills. The game should never penalize the group because of the misdoings of one player.

The game does not require the players to perform complex movements. The cognitive affordance changes slowly with each game level, but is low at the beginning. The only obstacles for the players to overcome are their impairments in social interaction and communication. Thus, the difficulty of the game lies in overcoming those impairments in collaborative behavior. The difficulty does not lie in solving cognitive tasks like in many other games. The more the players wish to collaborate, the more they will be rewarded.

Requirement 6: The difficulty of the game should be variable.

To keep the players motivated, they never need to be challenged too little or too much. They need to be in a constant state of flow, a "state of effortless concentration and enjoyment"[1]. 


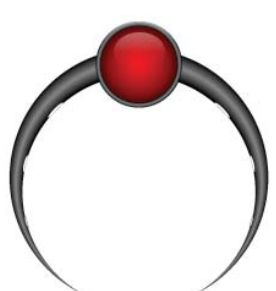

Figure 4. The "Neutralizer" catches a player and prevents him from shooting. If the player asks another shooting. If the player asks another
player for help, the neutralizer can be defeated very easily. Both communication on the part of the first player and collaborative behavior on the part of the second player are rewarded.

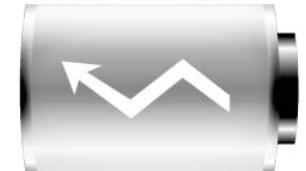

Figure 5. When a player runs out of enough energy to shoot, an "Energy Battery" appears on the other end of the screen. The player can then either reach for the battery or can ask another player to send the battery over to him. This is done by an easy swift gesture.

The second option is far more efficient and will lead to more points.
To achieve and maintain a state of flow, the difficulty should be variable. The difficulty of the game lies in overcoming impairments in collaborative behavior. The therapist can adjust the level of required minimal collaboration in the options menu. The higher this level is set, the more time is needed to eliminate the enemies, and the more the players need to cooperate.

The game also seeks to reward knowledge of the game and to surprise the player with new elements; players not only need to be in a state of "collaborative flow" (the game difficulty), but also in a state of "cognitive flow". This is achieved by offering them the choice of the game level, which affects the number of different enemy ships in the level. Since the elements of the game all encourage communication and social behavior, the therapeutic aspect is not influenced by the number of game elements. The higher the game level is set, the higher the cognitive affordance. By increasing this affordance, players stay motivated and curious. However, players who are new to the game should always start with a low cognitive affordance. This may lie within the responsibility of the therapist.

Therefore, the flow of the game is structured in two layers: the collaborative difficulty set by the therapist and the cognitive affordance set by the players. This "two-dimensional flow" allows for strong motivation and, at the same time, does not neglect the therapeutic effect.

Requirement 7: The length of the game should not exceed a timespan of ten minutes. The therapist must have the opportunity to reflect on the contents of the game together with the children to provide a transfer between the game and reality.
After choosing the desired game level, the game lasts three minutes. Taking possible explanation time into account, the estimated time needed to play is five minutes. After playing the game, the therapist should initiate a group discussion. The game provides a basis for such discussion. After completing game level, the game displays information regarding whether or not the players acted collaboratively and offers suggestions for improvement.

Requirement 8: Dominant behavior of a single player should be prevented. Every player must have the opportunity to integrate him-or herself in the process of the game.

Using cognitive tasks as gaming obstacles may lead to dominant behavior by a single player. When a cognitive task is solved by a single player, the other players will become a hindrance to him (for an example, see [3]). He may then apply dominant behavior to speed up the game process. As mentioned above, the main difficulty of this game does not lie in overcoming cognitive obstacles, but in performing collaborative actions. Dominant and uncooperative behavior is penalized and discouraged because it leads to a lower score.

\section{Expert Reviews}

The game was reviewed by a lecturer in game analysis and a professional designer of children's games as well as the therapist and one of the educational advisers with whom we generated the requirements. The aspects of encouraged collaboration and twodimensional flow were received positively. The structure and setup of the game were estimated to be easy to understand. 


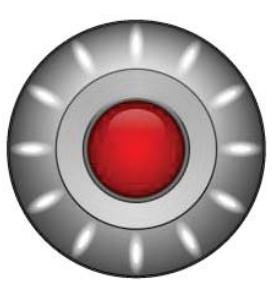

Figure 6. The "Teleporter" changes color and position. The player with the displayed color does significantly more damage to this enemy. Players who discuss their strategy will save time and gather more points.

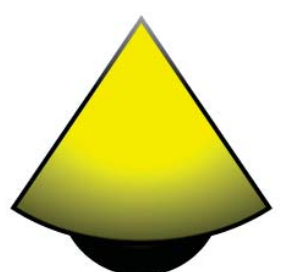

Figure 7. Players can collect the "Supply-Drone" by moving their token over it. By doing so, the players win additional time. Dividing tasks between the players will lead to a higher score.

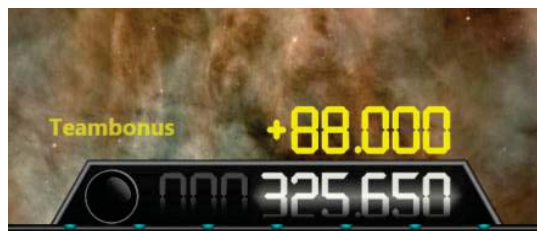

Figure 8. All elements encourage players into cooperative actions. Collaborative behavior is rewarded with additional points and is by no means enforced.
In its current form, the game can be used as a motivator and basis for discussion at the end of group therapy sessions.

However, while finding their requirements matched by the game, there were still issues for discussion and improvement. The number of enemy types can still be further developed. This would increase the need for discussing strategic issues. Motor requirements can also afford to be little higher.

\section{Conclusion and Future Work}

This work emphasizes the importance of encouraged collaboration in therapeutic games. Since therapeutic games have their roots in behavioral therapy, they should focus on the essence of such therapy.

While the game should always provide and maintain a level of motivation, the game needs to focus on its main therapeutic goal. This can be achieved through two-dimensional flow. The goal in this case is to enhance social interaction and communication. Similar to behavioral therapy, therapeutic games should encourage such behavior, but not enforce it. With these premises, therapeutic games can be more effective.

In future work, we will include our first findings to revise the game. We will then evaluate the game by testing it with groups of children in a treatment center for autism. Together with qualitative interviews conducted with the therapists, our observations will help us to validate the value of encouraged collaboration.

\section{Acknowledgements}

We would like to thank Andreas Wacker, Andreas

Targan, Katharina Lilje, and Steffen Bogen for sharing their experiences in generating the requirements and evaluating the game, and Vladislav Syomushkin for his help in modeling of the game tokens.

\section{References}

[1] Csíkszentmihályi, M. Finding flow: The Psychology of Engagement with Everyday Life. Basic Books, 1997.

[2] American Psychiatric Association. Diagnostic and Statistical Manual of Mental Disorders. 4th Ed.

Washington, DC, APA Pub, 2000.

[3] Piper, A.M., O'Brien, E., Morris, M.R., Winograd, T. SIDES: a cooperative tabletop computer game for social skills development. In Proc. CSCW 2006, ACM (2006), New York, NY, USA, 1-10.

[4] Magerkurth, C., Engelke, T., Memisoglu, M. Augmenting the virtual domain with physical and social elements: towards a paradigm shift in computer entertainment technology. Comput. Entertain. 2, 4 (October 2004), 12-12.

[5] Mesibov, G. Shea, V. Schopler, E. The TEACCH Approach to Autism Spectrum Disorders. Springer, 2004.

[6] Gal, E., Bauminger, N., Goren-Bar, D., Pianesi, F., Stock, O., Zancanaro, M., Weiss, P.L.: Enhancing Socia Communication of Children with High-Functioning Autism through a Co-located Interface. Artificial Intelligence \& Society 24, 75-84, 2009.

[7] Battochi, A., Ben-Sasson, A., Esposito, G., Gal, E., el al.: Calloborative Puzzle Game: A Tabletop Interface for Fostering Collaborative Skills in Children with Autism Spectrum Disorders. Journal of Assistive Technologies, 4(1), 4, 14, 2010. 